\title{
CARACTERIZACIÓN Y OPORTUNIDADES DE MEJORA DE LOS RESIDUOS SÓLIDOS EN UNA INSTITUCIÓN EDUCATIVA, LA ESPERANZA [TRUJILLO-PERÚ], 2019
}

\author{
CHARACTERIZATION AND OPPORTUNITIES FOR THE \\ IMPROVEMENT OF SOLID WASTE AT THE EDUCATIONAL \\ INSTITUTION, LA ESPERANZA
}

(TRUJILLO-PERÚ), 2019
Bilmia Veneros Urbina $^{1 *}$, (D) Paolo Amaya Alvarado², (D) Yaneth Aracelli Chuan Torres², (D) Cristhian Manchay Hernández ${ }^{2}$
bilmia_v@hotmail.com; paamalva@gmail.com; yacht86@hotmail.com; cmanchay79@gmail.com
1Universidad Nacional de Trujillo, Trujillo, Perú
¿Universidad César Vallejo, Lima, Perú

*Correspondencia: Bilmia Veneros Urbina. Email: bilmia_v@hotmail.com

Recibido: 01.07.2020 | Aprobado: 24.07.2020

\section{RESUMEN}

La investigación tuvo como propósito determinar la caracterización y oportunidades de mejora de los residuos sólidos en la institución educativa. La investigación es de tipo no experimental, presentó un diseño transversal y descriptivo. La población y muestra estuvo constituido por los residuos generados por los alumnos, personal docente y administrativo de la institución educativa San José del distrito de La Esperanza, provincia de Trujillo, región La Libertad, los cuales representan un total de 1551 personas. La medición y caracterización de los residuos se realizó tomando en cuenta la guía para la caracterización de los residuos sólidos domiciliarios, formulada por Kunitoshi Sakurai (1982). Las oportunidades de mejora, se elaboraron a través de un focus group y el brain storning donde participaron los integrantes de la comunidad educativa para luego ser mostrado a través de una matriz de doble entrada. Se determinó la caracterización de los residuos sólidos y se establecieron oportunidades de mejora que contribuyen a una adecuada gestión de residuos sólidos de la I.E "San José, La Esperanza - región La Libertad. Se caracterizó los residuos sólidos, siendo el residuo sólido con mayor porcentaje es papel con un $25,56 \%$, plástico con un $16,33 \%$ y restos de fruta con $15,69 \%$, mientras que los residuos con un menor porcentaje tales como metales con $2,08 \%$, cartón con un $5,26 \%$ y madera con un $5,26 \%$. La producción per cápita evidencia que cada persona puede generar residuos sólidos en un valor de 65 gramos por cada miembro de la comunidad educativa.

Palabras clave: Caracterización, oportunidades de mejora, residuos sólidos.

\section{ABSTRACT}

The purpose of the research was to determine the characterization and opportunities for improvement of solid waste at the educational institution. The research is non-experimental, presented a transversal and descriptive design. The population and sample consisted of the waste generated by the students, teaching and administrative staff of the San José educational institution of the district of La Esperanza, province of Trujillo, region La Libertad, which represent a total of 1551 people. The measurement and characterization of waste was carried out taking into account the guide for the characterization of household solid waste, formulated by Kunitoshi Sakurai (1982). The opportunities for improvement were developed through a focus group and brain storning where the members of the educational community participated, and then be shown through a double entry matrix. The characterization of solid waste was determined and improvement opportunities were established that contribute to an adequate solid waste management of the educational institution, La Esperanza - La Libertad region. Solid waste was characterized, the solid residue with the highest percentage being paper with $25.56 \%$, plastic with $16.33 \%$ and fruit remains with $15.69 \%$, while residues with a lower percentage such as metals with $2.08 \%$, cardboardwith $5.26 \%$ and wood with $5.26 \%$. Per capita production shows that each person can generate solid waste at a value of 65 grams for each member of the educational community.

Keywords: Characterization, improvement opportunities, solid waste. 


\section{INTRODUCCIÓN}

Los seres humanos han sido generadores de residuos sólidos desde las primeras formas de organización social alrededor de 10.000 AC (Worrell \& Vesilind, 2012). Históricamente, los problemas de salud pública, la seguridad, la escasez de recursos y el estado de las calles actuaron como factores impulsores de los sistemas de gestión de residuos (Louis, 2004; Melosi, 2005; Worrell \& Vesilind, 2012). Las pequeñas comunidades consiguieron enterrar los desechos sólidos en las afueras de sus asentamientos o disponer de ellos en los ríos cercanos o cuerpos de agua, pero a medida que la densidad de población aumentó, estas prácticas dejaron de ser eficientes, fueron la causa de la propagación de malos olores y enfermedades (Seadon, 2006).

Es por ello que resulta importante definir a los residuos sólidos, como los materiales o productos que se desechan, ya sea en estado sólido, semisólido, líquido o gaseoso, que se contienen en recipientes o depósitos, y que necesitan estar sujetos a tratamiento o disposición final o que viene a ser cualquier producto en estado gaseoso, líquido o sólido que se genera como consecuencia de las actividades humanas en procesos tales como la transformación, extracción o utilización y que tiene como destino ser desechado puesto que carece de valor para sus propietarios (Vértice, 2008).

De acuerdo a la Ley General de Residuos Sólidos 27314 (2000), los residuos sólidos vienen hacer sustancias, productos o subproductos que pueden producir un efecto negativo en la salud de las personas y el medio ambiente, por lo tanto, se requiere de un tratamiento adecuado mediante un sistema que incluya procesos en la reducción, clasificación, reutilización y almacenamiento de los residuos sólidos. Según Tchobanglous (1994) sostiene que los desperdicios sólidos son todos los excesos producto de las actividades humanas que terminan siendo eliminados porque resultan superfluos e inútiles. Otros autores definen a los residuos como cualquier material sobrante de las actividades industriales, comerciales y humanas que no tiene ningún valor residual (Ortiz, Pasqualino, Díez, \& Castells, 2010).

La Gestión Integral de Residuos Sólidos posee una ubicación importante dentro de la gestión ambiental, ya que constituye un tema de salud pública que involucra a diferentes autoridades y profesionales de nuestra sociedad (Marshall \& Farahbakhsh, 2013). Factores como el aumento de la población, cambios en el estilo de vida y en las formas de producción de los últimos años, han generado un incremento en la producción de residuos sólidos urbanos y como consecuencia de esto una composición cada vez más heterogénea (Pineda, 1998). Así mismo, el impacto ambiental generado por los sistemas de "GIRS" depende de la manera de manejar y disponer los residuos (Sales et al., 2006).

Asimismo, el manejo de residuos sólidos, describe a todas las actividades realizadas desde la generación hasta la disposición final de los mencionados, de manera técnica operativa que involucre la manipulación, acondicionamiento, almacenamiento temporal, transporte, tratamiento, disposición final y otros procesos (MINAM, 2016). Hace décadas, el sector de la recuperación apenas estaba regulado y únicamente se tomaba en cuenta el factor económico; por suerte, el progreso de nuestra sociedad ha ido acompañado por una mayor concienciación y cuidados del medio ambiente, y en ese enfoque ha avanzado la legislación. 
Para el Perú durante el 2012, únicamente el 38\% de los residuos sólidos municipales fueron dispuestos en rellenos sanitarios. Esta cifra muestra la deficiencia en el manejo de las más de 7.1 millones de toneladas de residuos que se generan en el Perú (MINEDU, 2019). La producción promedio a nivel nacional de residuos sólidos en el 2014, fue de 13 244 toneladas al día; mientras, las demás ciudades de la costa, sierra y selva produjeron 3 224, 2736 y 1314 toneladas al día respectivamente. (MINAM, 2015).

En el mundo el tema de fortalecimiento de la gestión integral de residuos sólidos en instituciones educativas también se ha estado trabajando con cierta ventaja respecto a los programas ambientales institucionales en Colombia, por lo indicado, países como Australia (CutterMackenzie, 2010), Egipto (Kandil, Abou Bakr, \& Mortensen, 2004) y Turquía (Kayihan \& Tönük, 2012) cuentan con programas ambientales institucionales con resultados exitosos en la disminución y optimización de residuos sólidos en instituciones educativas. Programas que involucran toda la comunidad educativa y poseen herramientas interactivas para fortalecer la educación ambiental, vinculando este tema con todas las áreas del conocimiento (Cutter-Mackenzie, 2010; Kandil et al., 2004; Kayihan \& Tönük, 2012).

Por ello, resulta trascendental tomar acción para evitar problemas sociales y ambientales. Esta situación afecta además a la población escolar dentro y fuera de las instituciones educativas (II.EE.). En este contexto, nace la necesidad de sensibilizar y formar a los y las estudiantes de todo el país en el adecuado manejo de los residuos sólidos. El cambio de hábitos en relación a la generación y manejo de residuos es un proceso que debe acompañarse de una metodología que permita dirigir los esfuerzos a la obtención de resultados permanentes. Solo de esta forma es posible implementar programas sostenibles, que logren transformarse en un sello característico de los miembros de la comunidad educativa (MINEDU, 2019).

La importancia del tema de la generación y manejo de los residuos no involucra solo los efectos ambientales y de salud pública derivados de su generación y manejo, también está implícito, desde otro ángulo, el uso de los recursos naturales. La gestión integral de los residuos, además de procurar reducir su generación y conseguir su adecuada disposición final, también puede dar como resultado colateral la reducción, tanto de la extracción de recursos (evitando su agotamiento), como de energía y agua que se utilizan para producirlos, así como la disminución de la emisión de gases de efecto invernadero. Todo ello se acompaña de importantes beneficios económicos, sociales y ambientales.

La investigación desarrollada nos evidencia la gestión de residuos sólidos en una institución educativa del distrito La Esperanza a nivel primario y secundario, esto gracias a la revisión de diversos instrumentos de gestión de residuos sólidos, tales como: plan de gestión ambiental de residuos sólidos, estudios de caracterización, entrevistas con la dirección y responsables inmediatos.

Las infraestructuras y áreas de disposición final de residuos sólidos también son un aspecto muy importante a tratar, ya que la institución educativa no cuenta con un lugar adecuado para el acopio de residuos sólidos porque el colegio carece de ese espacio determinado. Se presentará además el tipo de gestión en materia de reciclaje y manipulación de residuos que se realiza en una institución educativa del distrito de La 
Esperanza, cabe mencionar que también se evaluará si la gestión de residuos en la institución está implementada adecuadamente o solo es de carácter empírico.

Ante ello, fue formulado el problema: ¿Cuál es la caracterización y oportunidades de mejora de los residuos sólidos en la institución educativa?; asimismo, se propusieron como objetivo general: determinar la caracterización y oportunidades de mejora de los residuos sólidos en la institución educativa y como, específicos: caracterizar los residuos sólidos en la institución educativa y proponer oportunidades de mejora del manejo de los residuos sólidos en la institución educativa.

Por lo antes descrito, se precisa que la contribución científico y técnica de la investigación radica en establecer un mercado de reciclaje y potencial energético para futuras plantas de compostaje y/o producción de biogás, lo cual obligaría a emplear nuevas fuentes de generación de energía, presentando un alineamiento al desarrollo de energías renovables (Vásquez, 2011), siendo ello una tecnología necesaria para el aprovechamiento de los recursos, a pesar de presentar problemas financieros para su implementación.

Finalmente, se precisa que muchos estudios indican que estas formas de generación se vuelven cada vez más eficientes, económicas y autosostenibles para las diversas necesidades (confort, transporte, cocina, industria y electricidad), encontrándose alineados al modelo de las “3R" (reducción, reutilización y reciclaje), siendo una propuesta que busca construir una sociedad orientada hacia el reciclaje y específicamente da prioridad a la reducción en el volumen de residuos generados, y pretende desarrollar hábitos de consumo responsable (López, 2017).

\section{MATERIAL Y MÉTODOS}

La investigación es de tipo no experimental, presentó un diseño transversal y descriptivo. La población y muestra estuvo constituida por los residuos generados por los alumnos, personal docente y administrativo de la institución educativa del distrito de La Esperanza, provincia de Trujillo, región La Libertad, los cuales representan un total de 1551 personas.

\section{Área de Estudio}

El distrito de La Esperanza se encuentra ubicado, aproximadamente, a 4 kilómetros al norte del centro histórico de la provincia de Trujillo, en la región La Libertad, entre las coordenadas $8^{\circ} 04^{\prime} 59^{\prime \prime}$ de latitud Sur y $79^{\circ} 02^{\prime} 28^{\prime \prime}$ de longitud Oeste.

\section{Figura 1.}

Mapa de ubicación de la institución educativa - La Esperanza (Trujillo-Perú), 2019 


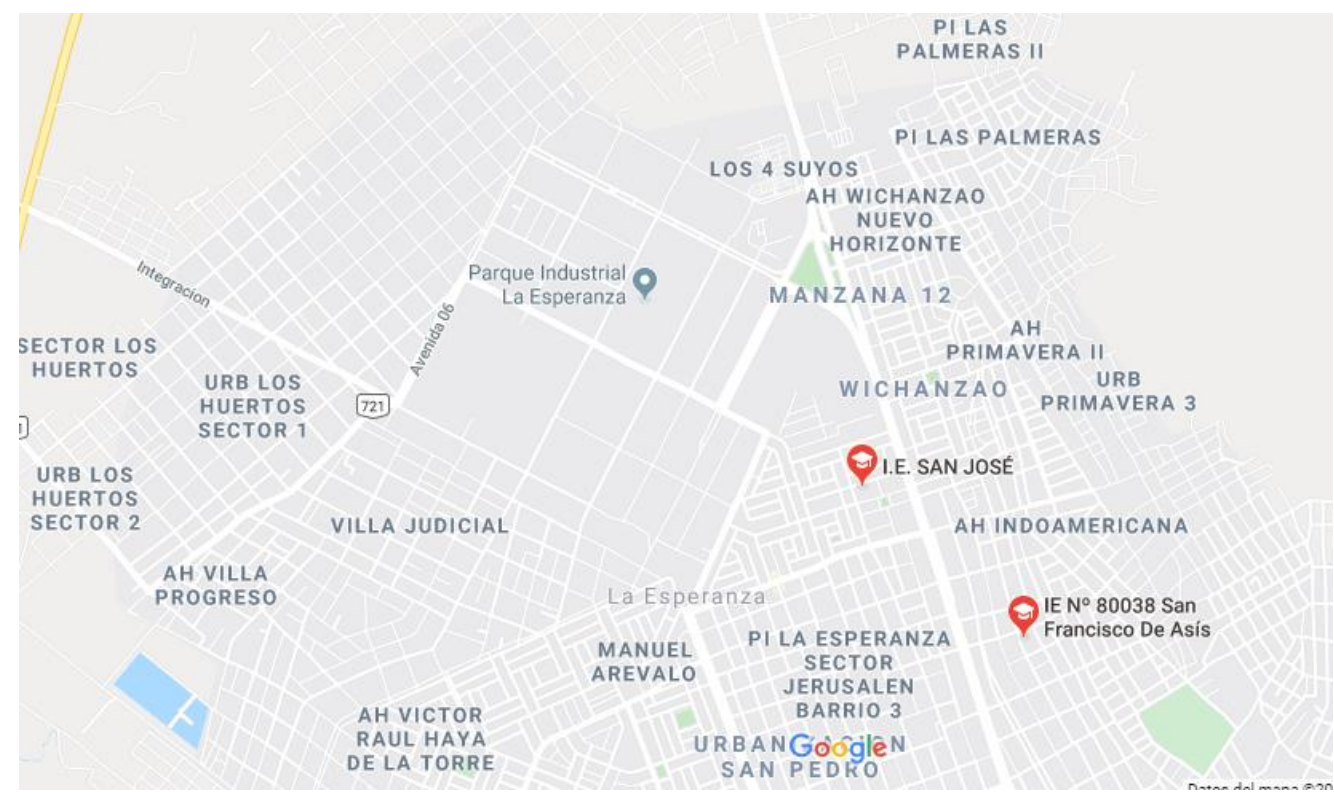

\section{Procedimiento}

- Se visitó la institución educativa en horario de clases, durante los 8 días que duró la caracterización, para observar el desarrollo de las actividades de la I.E., y el manejo de los residuos generados.

- Se realizaron entrevistas a un grupo representativo de la institución educativa, conformado por un personal de la dirección, un representante de los docentes, un representante de los alumnos, personal de limpieza, con quienes se analizó y priorizó la problemática del manejo actual de los residuos sólidos.

- Se recabó información por medio de la dirección de la institución educativa, al respecto de la infraestructura, número de estudiantes, trabajadores.

- Se observó el proceso de recolección de los residuos sólidos del plantel, por parte del personal de limpieza.

- Se tomó, como base para el diagnóstico, los resultados obtenidos en las encuestas y la caracterización de los residuos sólidos de la institución educativa.

- Se tomó registro fotográfico de las instalaciones de la institución educativa.

La medición y caracterización de los residuos se realizó tomando en cuenta la guía para la caracterización de los residuos sólidos domiciliarios, formulada por Kunitoshi Sakurai en1982 para el Centro Panamericano de Ingeniería Sanitaria y Ciencias del Ambiente (CEPIS), cuya metodología fue adaptada para el desarrollo de la investigación.

El muestreo se realizó durante 8 días útiles; una vez cada día al finalizar el horario escolar, se recolectaban las bolsas de residuos de todas las áreas del colegio, para ser trasladadas hacia el patio del colegio donde eran pesadas y caracterizadas de la siguiente manera: 
- Se colocó los residuos sobre un plástico grande, con la finalidad de no combinar los residuos con tierra.

- Se vertió el desecho formando un montón y se separaron los componentes, clasificándolos en: papel y cartón, restos orgánicos, plásticos, vidrio y otros (tierra, restos de losetas, madera, etc.)

- Los componentes se clasificaron y fueron llenadas en bolsas de 70 litros.

- Con ayuda de una balanza electrónica se pesaron las bolsas con los componentes y se registraron los pesos de los residuos.

- Para determinar la densidad se utilizó un contenedor cilíndrico con una capacidad de 65 litros, se midió la altura $(\mathrm{H})$ y el diámetro del contenedor y se registraron los datos, de acuerdo al formato de registro para determinar la densidad de los residuos.

- Se procedió a vaciar cada componente en el contendor, previa compactada a fin de eliminar espacios vacíos, luego se procedió a medir la altura libre del cilindro (h) que se refiere a la altura que se deja libre de residuos dentro de este, con la finalidad de calcular la altura útil del cilindro.

- Se realizó y registró este proceso durante los 8 días del estudio, descartándose al día uno, según las recomendaciones de la metodología del CEPIS, debido a que desconocíamos la existencia de basura de días anteriores a la primera toma de muestra, los siguientes cálculos se hicieron en base a los 7 días posteriores.

El periodo de monitoreo estuvo comprendido desde el lunes 21/10/2019 hasta el domingo 27/10/2019.

\section{Determinación del porcentaje por cada componente}

Se calculó el porcentaje de cada componente, teniendo en cuenta los datos del peso total de los residuos recolectados en un día (G) y el peso de cada componente (Gi).

$$
P s=[(G i / G) \times 100]
$$

\section{Donde:}

Ps $=$ Porcentaje del componente considerado; $\mathrm{Gi}=$ Peso del componente considerado; $G=$ Peso total de los residuos

\section{Determinación de la generación Per cápita}

Para el cálculo de la generación Per cápita de los residuos sólidos, se empleó el siguiente procedimiento: Se dividió el peso total de residuos ( $\mathrm{kg}$ ), generados en los 7 días, entre el número total de personas generadoras de residuos y se obtuvo la generación per cápita diaria promedio. Para realizar este cálculo se empleó la siguiente fórmula:

$$
\mathrm{Gpc}=[\mathrm{Wt} / \mathrm{Nt}]
$$

\section{Donde:}

$\mathrm{Gpc}=$ Generación per cápita $(\mathrm{kg} /$ persona/día); Wt $=$ Peso total de los residuos; $\mathrm{Nt}=$ Número total de personas 
Las oportunidades de mejora, se elaboraron a través de un focus group y el brain storning donde participaron integrantes de la comunidad educativa, para luego ser mostrado a través de una matriz de doble entrada.

\section{RESULTADOS Y DISCUSIÓN}

\section{Figura 2}

Expresión porcentual de residuos sólidos, según clasificación especifica, generados en la institución educativa, La Esperanza (Trujillo-Perú), 2019

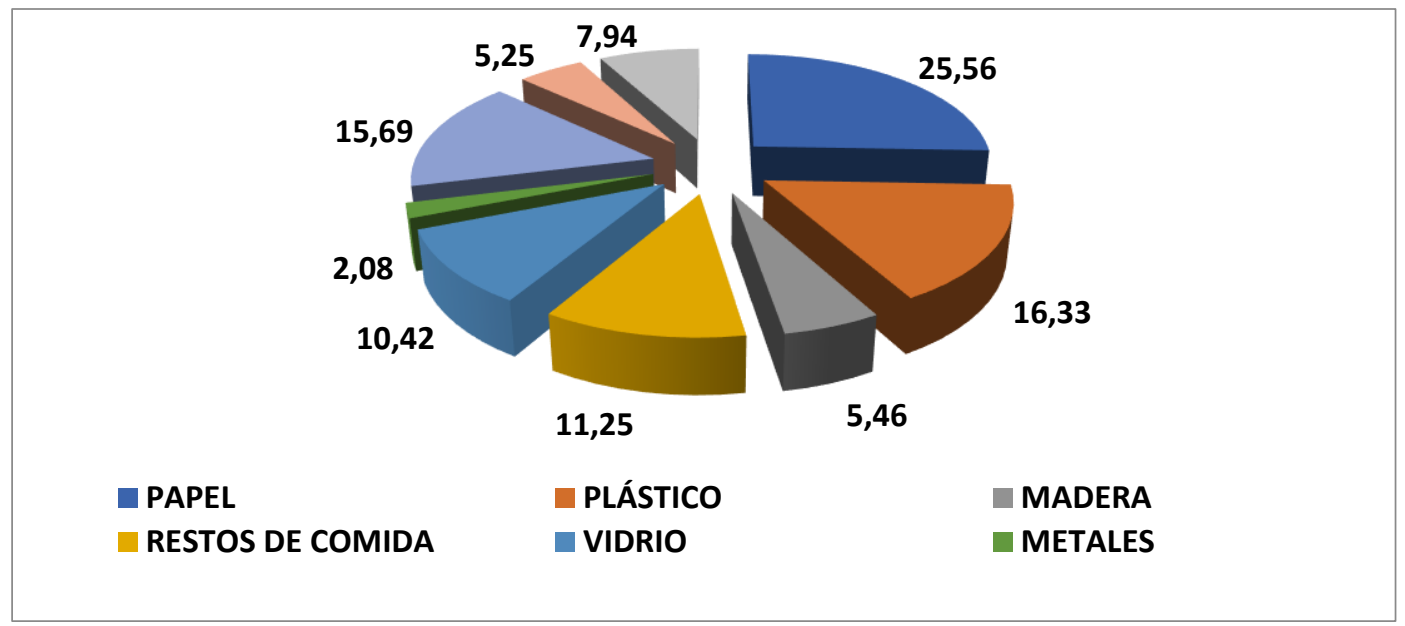

Se evidenció que los residuos sólidos con mayor porcentaje son papel con un $25,56 \%$, plástico con un $16,33 \%$ y restos de fruta con $15,69 \%$; mientras, los residuos con un menor porcentaje son los metales con $2,08 \%$, cartón con un $5,26 \%$ y madera con un $5,26 \%$.

\section{Figura 3}

Expresión porcentual de residuos sólidos, según clasificación general, generados en la institución educativa, La Esperanza (Trujillo-Perú), 2019

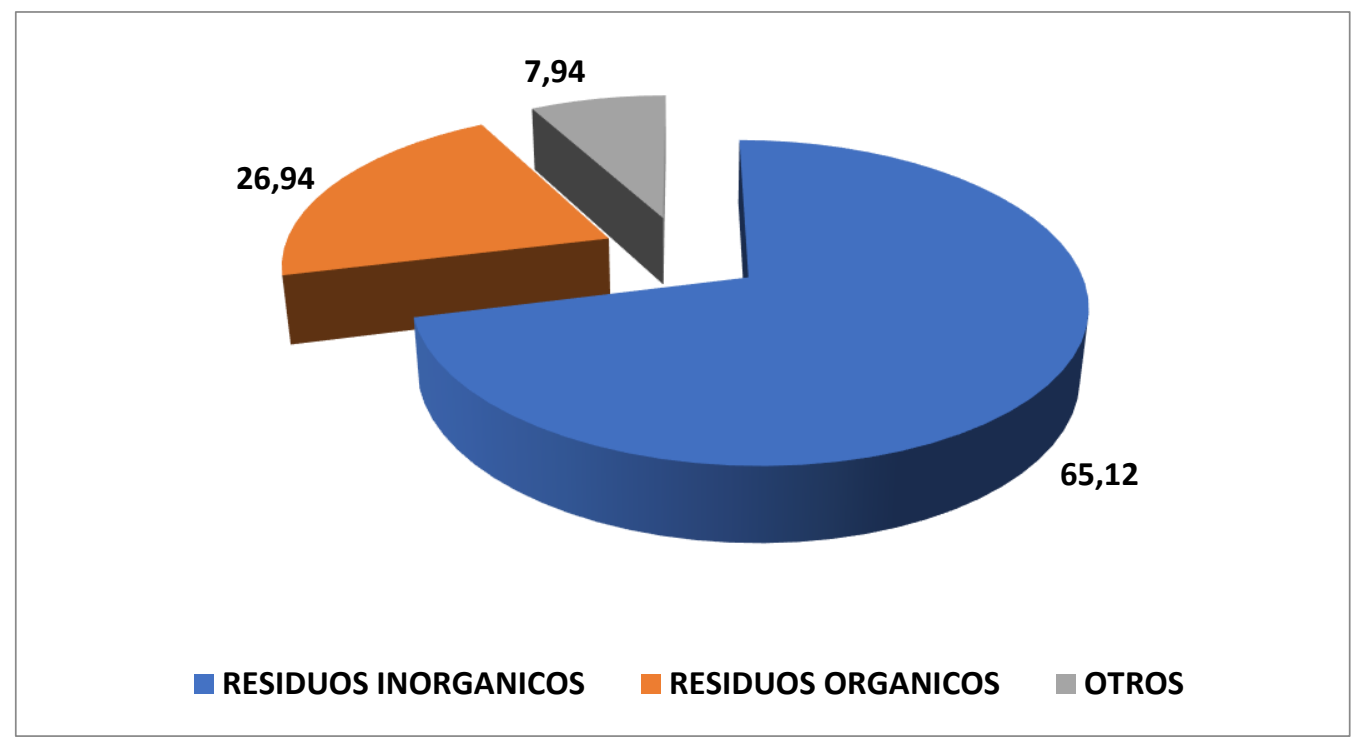

Se evidencia que el tipo de residuos que predominó en la institución educativa, participante de la investigación, fueron los residuos sólidos inorgánicos, los cuales 
alcanzaron un valor de $65,12 \%$ y los residuos orgánicos con un valor de $26,94 \%$. Todo ello se complementa, según los planes de manejo de residuos sólidos municipales y no municipales de los distritos de Los Olivos (2015) y El Rímac (2013) y el estudio de caracterización de residuos sólidos del distrito de Ate (2011), se estima que las instituciones educativas aportan entre el $2-6 \%$ al total de residuos generados en los distritos mencionados.

\section{Tabla 1}

Producción per cápita en la institución educativa, La Esperanza (Trujillo-Perú), 2019.

\begin{tabular}{|c|c|}
\hline Producción per cápita & $0,065 \mathrm{~kg} /$ persona \\
\hline
\end{tabular}

La producción per cápita evidencia que cada persona puede generar residuos sólidos en un valor de 65 gramos, por cada miembro de la institución educativa de La Esperanza, Trujillo - región La Libertad, 2019. Siendo un valor alineado por lo reportado en Quillos Ruiz et al. (2018) quienes obtuvieron una generación per cápita de RSD de 0,425 kg/hab/día, donde los RSOD representan el 69,03\% (0,297 kg/hab./día) de los residuos sólidos domiciliarios.

\section{Tabla 2.}

Matriz de oportunidades de mejora para la institución educativa, La Esperanza (Trujillo-Perú), 2019

\begin{tabular}{lll}
\hline Oportunidad de Mejor & Responsable & Tiempo \\
\hline Establecer una política de gestión ambiental & Dirección & Enero 2020 \\
para la I. E & Dirección & \\
$\begin{array}{l}\text { Definir un programa de capacitaciones en } \\
\text { temas ambientales }\end{array}$ & Fobrero 2020 \\
& Dirección & \\
Implementar el Comité ambiental & Docentes de CTA & Marzo 2020 \\
& Alumnos & \\
Implementar una Planta de Compostaje para & Dirección & Abril 2020 \\
la I.E. & Docentes de CTA & \\
Realizar un convenio con un operador de & Dirección & Marzo 2020 \\
residuos sólidos / asociación de recolectores & Docentes de CTA & \\
formales & Dirección & Junio/Noviembre \\
Implementar un programa de reciclatón & Docentes de CTA & 2020 \\
semestralmente & Alumnos & \\
\hline
\end{tabular}

Para la medición de la producción de residuos sólidos, se utilizó plantillas de Microsoft Excel dividida por dos caracteres. Este trabajo comprende como línea de investigación la gestión pública, al relacionar la gestión ambiental con la cantidad de residuos sólidos producida en la institución educativa.

Con los resultados obtenidos se rebeló que la producción per cápita de residuos sólidos por persona en la institución educativa San José de $0.065 \mathrm{~kg}$. por persona. Considerando que se labora de lunes a viernes, la producción semanal de estos residuos sólidos llega a un peso general de $100.73 \mathrm{~kg}$, lo cual es similar a lo obtenido por Quintero et al. (2003), en su investigación "Manejo de residuos sólidos en Instituciones Educativas"; 
se llegó a calcular de generación diaria de $10.5 \mathrm{Kg}$ y un peso volumétrico de 44.9101 $\mathrm{Kg} / \mathrm{m}^{3}$.

En tal sentido, SINIA (2013) señala que la generación per cápita (GPC) promedio de los residuos sólidos domiciliarios en el Perú es de 0,61 kilogramos/persona/día; de los cuales la generación per cápita (GPC) promedio en la región sierra es de 0.547 kilogramos/persona/día. Concluyendo que las instituciones deben contar con un sistema de clasificación de residuos sólidos que aplique un eficiente aporte y uso de estos residuos en la institución educativa.

Según Fuentes (2012) en su investigación "La significación de una economía ecológica radical", refuerza lo importante que es la relación entre la economía, la sociedad y la naturaleza, para velar por la calidad de vida de las personas, lo cual permite tomar como una contribución a la institución educativa San José y es coherente con la propuesta de oportunidades de mejora, antes planteadas para la institución educativa participante en la investigación.

\section{CONCLUSIONES}

La investigación concluye que:

Se determinó la caracterización de los residuos sólidos y se establecieron oportunidades de mejora que contribuyen a una adecuada gestión de residuos sólidos para la institución educativa del distrito de La Esperanza - región La Libertad.

Se caracterizó los residuos sólidos en la institución educativa, siendo el residuo sólido con mayor porcentaje el papel con un 25,56\%, plástico con un 16,33\% y restos de fruta con $15,69 \%$, mientras que los residuos con un menor porcentaje son metales con $2,08 \%$, cartón con un 5,26\% y madera con un 5,26\%.

La producción per cápita evidencia que cada persona puede generar residuos sólidos en un valor de 65 gramos, por cada miembro de la comunidad educativa de la I.E. del distrito de La Esperanza - región La Libertad, 2019.

Se elaboró una matriz donde se tiene las oportunidades de mejora para reducir y gestionar, adecuadamente, los residuos sólidos generados en la institución educativa del distrito de La Esperanza - región La Libertad, destacando la política de gestión ambiental, implementación de una planta de compostaje, programa de capacitaciones y una reciclatón, para ello se determinaron responsables y tiempos establecidos.

\section{REFERENCIAS BIBLIOGRÁFICAS}

Cutter-Mackenzie, A. (2010). Australian waste wise schools program: its past, present, and future. Journal of Environmental Education, 41, 165-178.

Collazos, H. (1997). Residuos Sólidos. Bogotá: Universidad Nacional.

Ley No 27314. (2000). Ley General de Residuos Sólidos Modificada por D.S No 1065. Ministerio del Ambiente.

Kandil, S. H., Abou Bakr, H., \& Mortensen, L. (2004). Incorporating Environmental Awareness of Solid Waste Management Within the Education System: (A Case From Egypt). Polymer-Plastics Technology and Engineering, 43(6), 1795-1803. 
Kayihan, K. S., \& Tönük, S. (2012). A study of litter and waste management policies at (primary) eco-schools in Istanbul. Waste Management \& Research, 30(1), 80-88.

López, M. (2017). Recogida y transporte de residuos urbanos o municipales. Madrid: Editorial CEP S.L.

Louis, G. E. (2004). A Historical Context of Municipal Solid Waste Management in the United States. Waste Management \& Research, 22(4), 306-322.

Marshall \& Farahbakhsh. (2013). Enfoques de sistemas para la gestión integrada de residuos sólidos en países en desarrollo.

Melosi, M. V. (2005). Garbage in the cities: refuse, reform, and the environment. Pittsburgh, Pa.: University of Pittsburgh Press

Ministerio del Ambiente. (2015). Guía metodológica para el desarrollo del Plan de Manejo de Residuos Sólidos. Ministerio del Ambiente, Perú.

Ministerio del Ambiente. (2016). Plan nacional de gestión integral de residuos sólidos 2016-2024.

MINEDU. (2019). Ministerio de Educación. Manejo de residuos sólidos en las Instituciones Educativas.

MUNICIPALIDAD DISTRITAL DE ATE. (2011). Estudio de caracterización física de residuos sólidos no domiciliarios del distrito de Ate. Página 64.

MUNICIPALIDAD DISTRITAL DE LOS OLIVOS. (2015). Plan distrital de manejo y gestión de residuos sólidos del distrito de los olivos.

MUNICIPALIDAD DISTRITAL DEL RIMAC. (2013). Plan de manejo de residuos sólidos del Rímac. Página (14).

Ortiz, O., Pasqualino, J., Díez, G., \& Castells, F. (2010). The environmental impact of the construction phase: An application to composite walls from a life cycle perspective. Resources Conservation and Recycling, 54(11):832-840.

PEAER. Plan Nacional de Desarrollo 2013-2018: El programa especial para el aprovechamiento de energías renovables.

Pineda, S. (1998). MANEJO Y DISPOSICION DE RESIDUOS SOLIDOS URBANOS. LIME-Acodal.

Quillos Ruiz, Serapio A., Escalante Espinoza, Nelver J., Sánchez Vaca, Daniel A., Quevedo Novoa, Luis G., \& De La Cruz Araujo, Ronal A. (2018). Residuos sólidos domiciliarios: caracterización y estimación energética para la ciudad de Chimbote. Revista de la Sociedad Química del Perú, 84(3), 322-335.

Quintero, B., Teutli, M., González, M., Ruiz, A., Jiménez, G. (2003) "Manejo de residuos sólidos en Instituciones Educativas". México.

Sales, M., Delerue-Matos, C., Martins, I., Serra, I., Silva, M., \& Morais, S. (2006). A waste management school appr oach towards sustainability. Resources consevation and recycling, 48, 197-207. 
Seadon, J. K. (2006). Integrated waste management - Looking beyond the solid waste horizon. Waste Management, 26(12), 1327-1336.

SINIA. (2013). Generación per cápita de residuos sólidos. Ministerio del Ambiente: Biblioteca nacional del Perú.

Tchobanoglous, G. (1994). Gestión integral de residuos sólidos. Madrid: McGraw-Hill.

Vásquez OC. Gestión de los residuos sólidos municipales en la ciudad del Gran Santiago de Chile: Desafíos y oportunidades. Rev Int Contam Ambie. 2011; 27(4): 347-355

Vértice, P. (2008). Gestión medioambiental: manipulación de residuos y productos químicos. Málaga.

Worrell, W. A., \& Vesilind, P. A. (2012). Solid waste engineering. Australia; Stamford, CT: Cengage Learning.

\section{CITAR COMO:}

Veneros Urbina, B., Amaya Alvarado, P., Chuan Torres, Y. A., \& Manchay Hernández, C. (2020).

Caracterización y oportunidades de mejora de los residuos sólidos en una institución educativa, La Esperanza (Trujillo-Perú), 2019. Puriq, 2(3), 222-232. https://doi.org/10.37073/puriq.2.3.96 